\title{
Silica Microfibres for Broadband Third Harmonic Generation
}

\author{
Timothy Lee, ${ }^{1 *}$ Yongmin Jung, ${ }^{1}$ Christophe A. Codemard, ${ }^{2}$ \\ Ming Ding, ${ }^{1}$ Neil G. R. Broderick, ${ }^{3}$ and Gilberto Brambilla ${ }^{1}$ \\ ${ }^{1}$ Optoelectronics Research Centre, University of Southampton, Southampton, SO17 1BJ, United Kingdom. \\ ${ }^{2}$ SPI Lasers, Southampton, SO30 2QU, United Kingdom. \\ ${ }^{3}$ Physics Department, University of Auckland, Private Bag 92019, Auckland, New Zealand. \\ *Corresponding author: tl305@orc.soton.ac.uk
}

\begin{abstract}
We report broadband third harmonic generation via intermodal phase matching in silica microfibres of several centimetres. A $5 \mathrm{~dB}$ conversion bandwidth exceeding $36 \mathrm{~nm}$ was recorded, with harmonic power detected over a $150 \mathrm{~nm}$ range.
\end{abstract}

(C) 2011 Optical Society of America

OCIS codes: (190.0190) Nonlinear Optics; (190.2620) Harmonic generation and mixing; (190.4370) Nonlinear optics, fibers

Recent interest in the use of microfibres for third harmonic generation (THG) [1-3] has been driven by their high nonlinearity [4] which arises from their wavelength-scale diameter $D$ and strong refractive index contrast. In particular, efficient THG is possible by phase matching the fundamental pump $H E_{11}(\omega)$ mode with a higher order third harmonic mode which experiences the same effective index [1], thus using intermodal dispersion to compensate for material and waveguide dispersion. However, this phase matching condition is only satisfied for certain microfibre diameters $D$ which limits the bandwidth of a uniform taper [1]. Here, we discuss the fabrication and characterisation of long silica tapers with lengths $L>3 \mathrm{~cm}$ in which the spread of diameters in the transition regions is utilised to collectively allow phase matching over a far wider range of pump frequencies. This technique is analogous to the use of chirping in quasi phase matched devices to extend their bandwidth [5], and offers the potential for broad third harmonic continua to be generated in the green down to the UV wavelengths for diverse applications such as biological spectroscopy.

Firstly, the critical phase matching diameters were calculated by solving the rigorous modal eigenvalue equations of a step index profile microfibre [6]. Fig. 1(a) plots the predicted phase matching wavelength at different diameters for $v=1,3$ hybrid TH modes. Since the experimental tapers were fabricated with diameters around $2 \mu \mathrm{m}$, a 1.55 $\mu \mathrm{m}$ pump will phase match to several modes at different points on the transition region. The overlap integral $J_{3}$ [1]
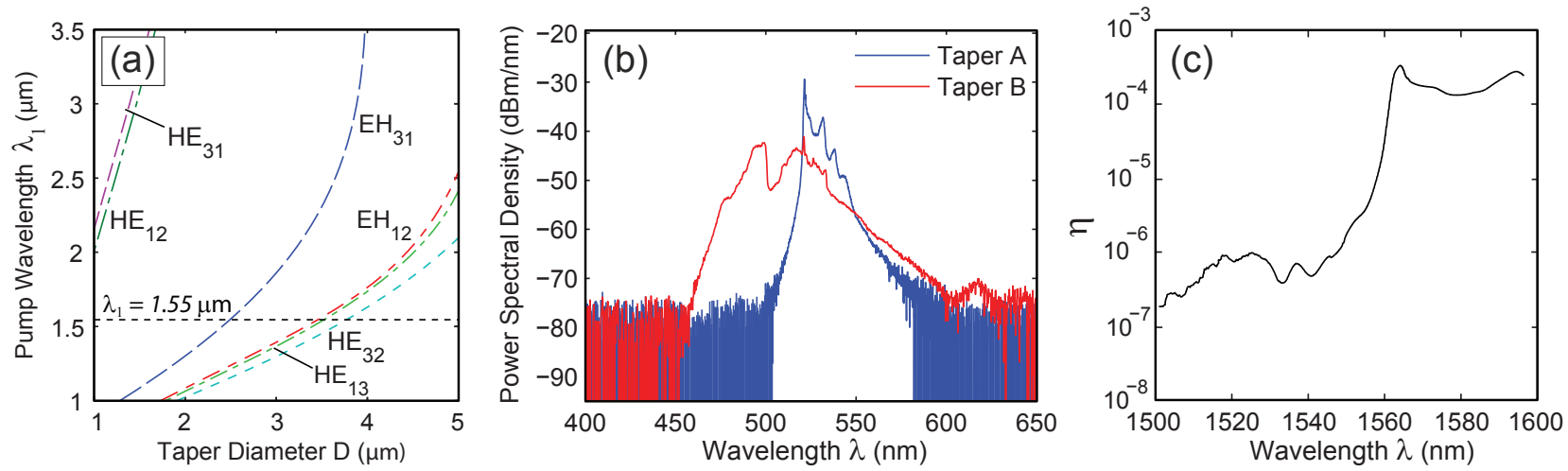

Fig. 1. (a) Predicted phase matching diameter against pump wavelength. (b) Third harmonic spectra when $P_{p}=1.25 \mathrm{~kW}$ for taper A $(D=2.1 \mu \mathrm{m}, L=45 \mathrm{~mm})$ and taper B $(D=1.8 \mu \mathrm{m}, L=45 \mathrm{~mm})$, where length $L$ includes the transition regions. (c) Conversion efficiency for taper A. 
between these TH modes and the pump mode are all similar and reside within the range $10^{-4}<J_{3}<10^{-3} \mu m^{-2}$ ) and so the third harmonic output signal is expected to contain a significant fraction of power from each mode.

Tapers were fabricated from standard single mode fibre using the modified flame brushing method [7] in which the tensioned fibre is translated back and forth through a stationary ceramic heater at $1320^{\circ} \mathrm{C}$. During pulling, the fibre was pumped with $4 \mathrm{~ns} 1.55 \mu \mathrm{m} 100 \mathrm{kHz}$ pulses with average and peak powers of $P_{\mathrm{av}}=0.5 \mathrm{~W}$ and $P_{p}=1.25 \mathrm{~kW}$ respectively. The taper output was passed through a shortpass filter with a $1300 \mathrm{~nm}$ cutoff wavelength, before being monitored on a spectrum analyser such that the onset of THG can be observed and the rig stopped accordingly.

Examples of third harmonic spectra from two tapers are shown in Fig. 1(b). As the waist narrowed from $3.3 \mu \mathrm{m}$ to $2.4 \mu \mathrm{m}$ during pulling, the $\mathrm{TH}$ peak grew by $25 \mathrm{~dB}$ whilst simultaneously falling from $600 \mathrm{~nm}$ to $520 \mathrm{~nm}$ as expected from Fig. 1(a). Furthermore, the TH signal remains even when the waist is several hundred nanometres narrower than the critical value, implying that the THG occurs primarily in the transition regions. Initially, the pump pulses' full-width half-maximum linewidth is narrowband $(<0.5 \mathrm{~nm})$, but nonlinearly broadens to several hundred nanometres whilst propagating through the taper. These broadened frequency components are then phase matched along the waist/uptaper resulting in the wide THG spectra.

For taper A, the TH spectrum shows not only the peak at $522 \mathrm{~nm}$, but also the third harmonic of the secondary peaks from broadened pump frequencies at longer wavelengths. When the pump peak power was increased from 0.9 $\mathrm{kW}$ up to $1.3 \mathrm{~kW}$, the upper limit of the detected TH spectral range increased from $540 \mathrm{~nm}$ up to $570 \mathrm{~nm}$. Note that the components of the pump which broadened to lower frequencies can phase match in the transition regions, but the higher frequencies require a phase matching diameter narrower than the taper waist and hence cannot generate a detectable TH signal. For this reason, the TH spectrum is asymmetric and skewed towards longer wavelengths.

Calculating the efficiency $\eta$ over moving $5 \mathrm{~nm}$ steps at maximum pump power (10 $\mu \mathrm{J}$ pulse energy) provides the conversion rates shown in Fig. 1(c). At $\lambda=1550 \mathrm{~nm}$, the value of $\eta=10^{-6}$ is low but nonetheless comparable to the efficiency reported in past experiments with uniform diameter waveguides [3]. For shorter pump wavelengths, $\eta$ is close to $10^{-7}$ since the THG is not phase matched. However, for $\lambda>1560 \mathrm{~nm}$ the efficiency is over $10^{2}$ times greater at $0.3 \times 10^{-3}$, with a $5 \mathrm{~dB}$ bandwidth of at least $36 \mathrm{~nm}$. The true bandwidth is likely greater, but confirmation would require a longer wavelength source and detector.

To further illustrate the effect of the taper profile on the third harmonic spectra, taper B was fabricated with a narrower waist of $D=1.8 \mu \mathrm{m}$. From its output spectrum in Fig. 1(b), the third harmonic signal is twice as wide as that recorded from taper A and covers a range of $150 \mathrm{~nm}$ at $P_{p}=1.25 \mathrm{~kW}$. Since the shorter wavelength components of the pump are now also able to phase match, the TH spectrum therefore appears more symmetrical and extends down to $450 \mathrm{~nm}$ (60 nm lower than for taper A). Compared to the THG in uniform waveguides in [2], the spectral width is an order of magnitude broader despite pumping at a tenth of the peak power. However, it remains important to note the bandwidth-efficiency trade-off: since the third harmonic generation is occurring in a steeper section of the transition region due to the narrower waist, the efficiency of $\eta=5 \times 10^{-5}$ is much lower compared to taper A.

In conclusion, we have experimentally demonstrated that THG in tapered optical microfibres several centimetres long with extended transition regions can exhibit a wider bandwidth than uniform waveguides. Silica microfibres were fabricated with diameters close to the phase matching diameters and lengths up to $45 \mathrm{~mm}$, and characterisation using a $1.55 \mu \mathrm{m}$ pump revealed third harmonic signals detected over a wavelength range exceeding $150 \mathrm{~nm}$ with efficiencies in the range $10^{-6}<\eta<0.3 \times 10^{-3}$ and a $5 \mathrm{~dB}$ bandwidth of at least $36 \mathrm{~nm}$.

\section{References}

1. V. Grubsky \& A. Savchenko, "Glass micro-fibers for efficient third harmonic generation," Opt. Express 13, 6798-6806 (2005).

2. D. Akimov et al. "Generation of a spectrally asymmetric third harmonic with unamplified $30-\mathrm{fs}$ Cr: forsterite laser pulses in a tapered fiber," Appl. Phys. B 76, 515-519 (2003).

3. V. Grubsky \& J. Feinberg, "Phase-matched third-harmonic UV generation using low-order modes in a glass micro-fiber," Opt. Commun. 274, 447-450 (2007).

4. S. Afshar \& T. M. Monro, "A full vectorial model for pulse propagation in emerging waveguides with subwavelength structures part I: Kerr nonlinearity," Opt. Express 17, 2298-2318 (2009).

5. M. A. Arbore, A. Galvanauskas, D. Harter, M. H. Chou. \& M. M. Fejer, "Engineerable compression of ultrashort pulses by use of secondharmonic generation in chirped-period-poled lithium niobate," Opt. Lett. 22, 1341-1343 (1997).

6. A. Snyder \& J. Love, "Optical Waveguide Theory," 1st ed., Springer, (1983).

7. G. Brambilla et al., "Optical fiber nanowires and microwires: fabrication and applications," Advances in Optics and Photonics 1, 107-161 (2009). 